\title{
The rich inner world of colorado potato beetles - a metagenomic survey of viral diversity in public data
}

\author{
Maria Starchevskaya \\ SRC VB "Vector" Rospotrebnadzor, \\ Koltsovo, Russia \\ starchevskayamaria@mail.ru
}

\author{
Yuri Vyatkin \\ Novosibirsk State University, \\ Novosibirsk, Russia \\ Novel Software Systems LLC, \\ Novosibirsk, Russia \\ vyatkin@gmail.com
}

\author{
Denis Antonets \\ SRC VB "Vector" Rospotrebnadzor, \\ Koltsovo, Russia \\ Novel Software Systems LLC, \\ Novosibirsk, Russia \\ antonec@yandex.ru
}

\begin{abstract}
Recent metagenomic studies of various environments revealed an enormous number of viruses, with hundreds of previously unknown species and even new virus families, and thus greatly expanded our understanding of the virosphere. Investigations of insect virome will provide new insights into ecology and evolution of viruses and their hosts. Colorado potato beetle is one of the most serious insect pests feeding on Solanaceae plants. The Colorado potato beetle virome has not been studied yet and, as far as we know, our work is the first attempt to discover viral material in DNA- and RNA-Seq data of $L$. decemlineata. We also hope that identification of new viruses will help to extend the arsenal of biopesticides.
\end{abstract}

Keywords - Colorado potato beetle, metagenomic analysis, virome, insect viruses

\section{Motivation and aim}

Colorado potato beetle (Leptinotarsa decemlineata from Chrysomelidae family) is one of the most serious insect pests feeding on Solanaceae plants. Originated from North America it became widespread worldwide and poses a significant threat to potato crops [1]. Besides, leaf feeding beetles transmit plant viruses [2,3]. Currently, new biological strategies to control the leaf feeding beetles are actively being developed [4-6], but all of them are based on either entomopathogenic fungi or bacteria, not viruses. However, entomopathogenic viruses are successfully used against Lepidoptera pests for decades $[7,8]$. The Colorado potato beetle virome has not been studied yet and, as far as we know, our work is the first attempt to discover the viral material in DNA- and RNA-Seq data of $L$. decemlineata. We also hope that identification of new viruses will help to extend the arsenal of biopesticides.

The main goal of this work was the identification of viral sequences from publicly available $L$. decemlineata transcriptomic and genomic samples.

\section{Methods}

L. decemlineata transcriptomic and genomic samples were taken from NCBI SRA. To minimize contamination the reads were filtered against UniVec database, containing the sequences of commonly used vectors, adapters, linkers, and primers, and also against human genome (GRCh38). The reads that were not aligned to $L$. decemlineata genome (GCA_000500325.2, Ldec_2.0,640 Mbp) were assembled de novo with MetaSPAdes software [9]. Contigs with sufficiently high coverage were analyzed with BLAST and MetaGeneMark software [10]. The reads not aligned to reference genome were aligned to viral genomes extracted from NCBI RefSeq. Non-aligned reads and contigs were also analyzed with Kraken2 software [11].
Results

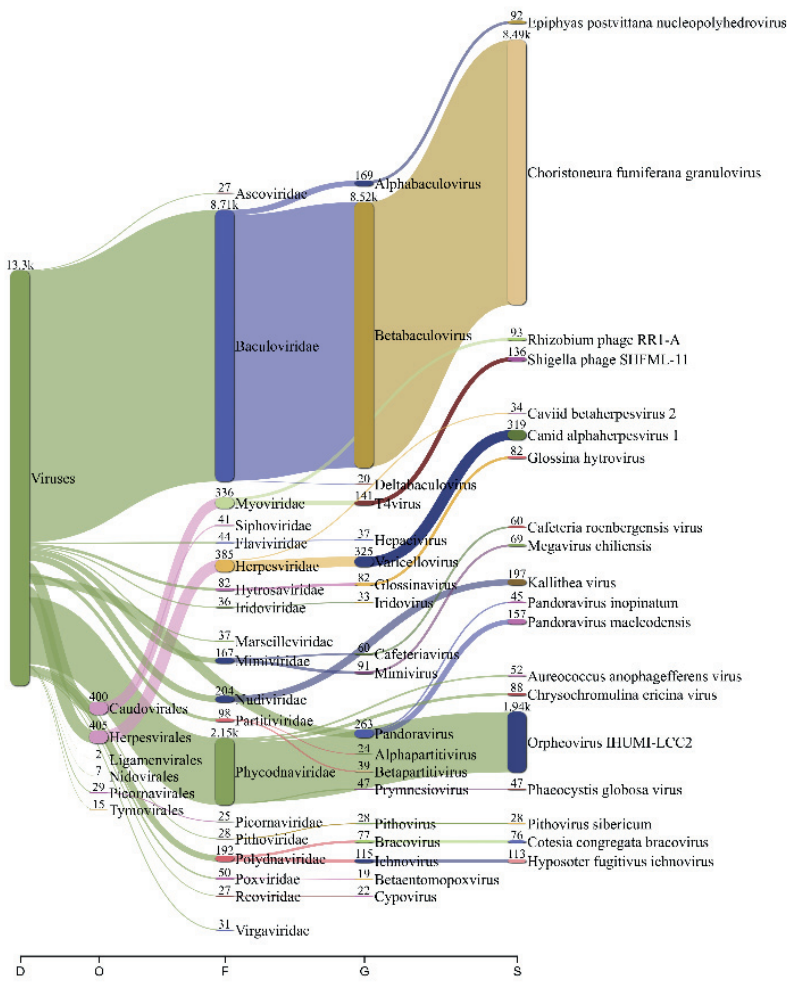

Fig. 1. Classification of reads from pooled RNA-Seq samples from L. decemlineata heads. The analysis was performed with Kraken2 [11]. The classification results were visualized using Pavian [12]. Only viral reads are shown

As it was expected, the most frequently found sequences belonged to common insect viruses of Iridoviridae, Baculoviridae and Iflaviridae families (Fig. 1). We were able to find whole genome sequences of several plant viruses: Potato virus S, Potato virus Y, Grapevine virus, Ligustrum virus. It was found that some genes predicted with MetaGeneMark probably belonged to Bracovirus genus. Further examination of Colorado potato beetle reference genome revealed numerous long fragments of Bracovirus genomic sequences (over 25,000 bp in total). Bracoviruses belong to Polydnaviridae virus family associated with parasitoid wasps. This is a very interesting family of viruses as they help to suppress the immune responses and alter the growth, the metabolism and endocrine system balance of wasp hosts [13]. Bracoviruses are also involved into horizontal gene transfer and they were shown to integrate into genomes of wasp hosts [14]. We analyzed 18 more Coleoptera genomes and found that Colorado potato beetle genome was the fourth most enriched with bracoviral sequences. As far as we know, 
this is the first report of a polydnavirus found in Chrysomelidae species. However, this fact is not surprising as there is a wasp Edovium puttleri known to parasitize on Colorado potato beetle eggs [15] and it was even proposed as a potential biocontrol agent. We have also found a number of long contigs with relatively high reads coverage that we couldn't identify yet.

\section{REFERENCES}

[1] Krawczyk K. et al. (2015) Prevalence of Endosymbionts in Polish Populations of Leptinotarsa decemlineata (Coleoptera: Chrysomelidae), J. Insect Sci., 15:106.

[2] Wickizer S.L., Gergerich R.C. (2007) First Report of Japanese Beetle (Popillia japonica) as a Vector of Southern bean mosaic virus and Bean pod mottle virus, Plant Dis., 91:637-637.

[3] Kersch-Becker M.F., Thaler J.S. (2014) Virus strains differentially induce plant susceptibility to aphid vectors and chewing herbivores, Oecologia, 174:883-892.

[4] Charpentier G. et al. (2002) Characterization of cell lines developed from the Colorado potato beetle, Leptinotarsa decemlineata Say (Coleoptera: Chrysomelidae), In Vitro Cell. Dev. Biol. Anim., 38:7378.

[5] Martin P.A.W. et al. (2007) Toxicity of Chromobacterium subtsugae to southern green stink bug (Heteroptera: Pentatomidae) and corn rootworm (Coleoptera: Chrysomelidae), J. Econ. Entomol., 100:680684.
[6] Yu Y. et al. (2016) Construction of an environmental safe Bacillus thuringiensis engineered strain against Coleoptera, Appl. Microbiol. Biotechnol., 100:4027-4034.

[7] Cuartas P.E. et al. (2019) Novel biopesticide based on Erinnyis ello betabaculovirus: characterization and preliminary field evaluation to control Erinnyis ello in rubber plantations, Pest Manag. Sci., 75:13911399.

[8] Moore S. et al. (2015) The Cryptophlebia Leucotreta Granulovirus10 Years of Commercial Field Use, Viruses, 7:1284-1312.

[9] Bankevich A. et al. (2012) SPAdes: A New Genome Assembly Algorithm and Its Applications to Single-Cell Sequencing, J. Comput. Biol., 19:455-477.

[10] Zhu W. et al. (2010) Ab initio gene identification in metagenomic sequences, Nucleic Acids Res., 38:e132.

[11] Wood D.E., Salzberg S.L. (2014) Kraken: ultrafast metagenomic sequence classification using exact alignments, Genome Biol., 15:R46.

[12] Ye X. et al. (2018) Parasitoid polydnaviruses and immune interaction with secondary hosts, Dev. Comp. Immunol., 83:124-129.

[13] Strand M.R., Burke G.R. (2015) Polydnaviruses: From discovery to current insights, Virology, 479-480:393-402.

[14] Lashomb J. et al. (1987) Edovum puttleri (Hymenoptera: Eulophidae), an Egg Parasitoid of Colorado Potato Beetle (Coleoptera: Chrysomelidae): Development and Parasitism on Eggplant, J. Econ. Entomol., 80:65-68. 